\title{
A single ultrasensitive assay for detection and discrimination of tau aggregates of Alzheimer and Pick diseases
}

\author{
Michael A. Metrick II ${ }^{1,2}$, Natália do Carmo Ferreira' ${ }^{1}$, Eri Saijo ${ }^{1}$, Allison Kraus ${ }^{1}$, Kathy Newell ${ }^{3}$, Gianluigi Zanusso ${ }^{4}$, \\ Michele Vendruscolo ${ }^{2}$, Bernardino Ghetti ${ }^{3}$ and Byron Caughey ${ }^{1 *}$ (D)
}

\begin{abstract}
Multiple neurodegenerative diseases are characterized by aggregation of tau molecules. Adult humans express six isoforms of tau that contain either 3 or 4 microtubule binding repeats ( $3 R$ or $4 R$ tau). Different diseases involve preferential aggregation of 3R (e.g Pick disease), 4R (e.g. progressive supranuclear palsy), or both 3R and 4R tau molecules [e.g. Alzheimer disease and chronic traumatic encephalopathy]. Three ultrasensitive cell-free seed amplification assays [called tau real-time quaking induced conversion (tau RT-QulC) assays] have been developed that preferentially detect $3 R$, 4R, or $3 R / 4 R$ tau aggregates in biospecimens. In these reactions, low-fg amounts of a given self-propagating protein aggregate (the seed) are incubated with a vast excess of recombinant tau monomers (the substrate) in multi-well plates. Over time, the seeds incorporate the substrate to grow into amyloids that can then be detected using thioflavin T fluorescence. Here we describe a tau RT-QulC assay (K12 RT-QulC) that, using a C-terminally extended recombinant 3R tau substrate (K12CFh), enables sensitive detection of Pick disease, Alzheimer disease, and chronic traumatic encephalopathy seeds in brain homogenates. The discrimination of Pick disease from Alzheimer disease and chronic traumatic encephalopathy cases is then achieved through the quantitative differences in K12 RT-QulC assay thioflavin T responses, which correlate with structural properties of the reaction products. In particular, Fourier transform infrared spectroscopy analysis of the respective K12CFh amyloids showed distinct $\beta$-sheet conformations, suggesting at least partial propagation of the original seed conformations in vitro. Thus, K12 RT-QulC provides a single assay for ultrasensitive detection and discrimination of tau aggregates comprised mainly of $3 R$, or both $3 R$ and $4 R$, tau isoforms.
\end{abstract}

Keywords: RT-QulC, Tau, Pick disease, Alzheimer disease, Chronic traumatic encephalopathy, Neurodegeneration, Protein misfolding, Seed, Primary age-related tauopathy

\section{Introduction}

Recent calls for better biomarkers of neurodegenerative diseases [20] have elicited efforts to detect disease-associated protein aggregates in diagnostically-accessible tissues. Among the novel approaches employed are those exploiting the self-propagating property of amyloid or pre-amyloid aggregates, that is, the ability to act as seeds that can grow by incorporating more monomers of their constituent proteins by seeded polymerization. Seeding activities in biospecimens from disease cases can be amplified by many orders of magnitude in vitro by using recombinant protein monomers as

\footnotetext{
* Correspondence: bcaughey@nih.gov

'LPVD, Rocky Mountain Laboratories, NIAID, NIH, Hamilton, MT 59840, USA Full list of author information is available at the end of the article
}

substrates and either sonication [1] or shaking [3, 9] to accelerate polymerization. The formation of amplified amyloid products in such reactions can be detected over time in multi-well plates using the amyloid-sensitive dye thioflavin $\mathrm{T}$ (ThT) $[9,33]$. For example, real time quaking-induced conversion (RT-QuIC) assays have been adapted to the ultrasensitive detection of prions $[2,18,24-26,32,33]$ as well as pathologic forms of $\alpha$-synuclein $[4,8,13,19,30]$ and tau [21, $23,28]$. These assays are providing a basis for accurate molecular diagnosis of the associated neurodegenerative diseases $[5,18,35]$.

Diseases involving tau pathology include those with preferential aggregation of 3 microtubule binding-repeat (3R), 4-repeat (4R), or both $3 R$ and $4 R(3 R / 4 R)$ tau 
isoforms. Previously, our lab has developed tau RT-QuIC assays optimized to detect the mainly $3 \mathrm{R}$ aggregates of Pick disease (PiD) [27]; the 3R/4R aggregates of Alzheimer disease $(\mathrm{AD})$ and chronic traumatic encephalopathy (CTE) [21]; or the 4R aggregates of progressive supranuclear palsy (PSP), corticobasal degeneration (CBD), frontotemporal dementias associated with MAPT mutations (FTDP 17 MAPT) and others [29]. In these assays, tau seeds from crude brain homogenates or cerebrospinal fluid (CSF) are added to reaction mixtures containing purified tau fragments truncated at different points in the microtubule binding domains to help confer seeding selectivity. For instance, the modified 3R tau fragment K19CFh forms fibrils when seeded with PiD brain homogenate as dilute as $10^{-9}$, but not with PSP brain homogenate even at a $10^{5}$-fold higher concentration [27]. In contrast, the modified 4R tau fragment K18CFh amplifies $4 \mathrm{R}$ tau aggregates [29], and a tau fragment spanning the cross- $\beta$ core of AD tau fibrils ( $\tau 306-378)$ selectively amplifies mixed 3R/4R tau seeds [21]. Figure 1 schematically represents the recombinant tau fragments used in different tau RT-QuIC assays.

Recently, structures of tau filaments of $\mathrm{AD}, \mathrm{CTE}$, and PiD have been solved by cryo-electron microscopy [14-16, 36]. These structures reveal that the $3 \mathrm{R}$ tau filaments characteristic of PiD differ in conformation from the 3R/4R filaments of $\mathrm{AD}$ and CTE. Since we previously achieved selective amplification of 3R PiD seeds with K19CFh, and amplification of
3R/4R AD and CTE seeds with $\tau 306-378$, we investigated whether a hybrid tau fragment could be seeded by PiD, AD, and CTE tau aggregates. We chose an extended 3R substrate that included amyloid core residues of both the PiD and $\mathrm{AD}$ tau filaments. This tau fragment, henceforth referred to as K12CFh (K12 cysteine-free and histidine tagged), spans the first, third and fourth repeats (R1, R3 and R4, respectively), extends to residue 400 , and is a modification of the previously described K12 construct [34]. Our new tau assay, K12 RT-QuIC, detects 3R and 3R/4R tau seeds with similar sensitivity while at the same time discriminating $\mathrm{PiD}$ seeds from the others based on conformational differences between the amplified RT-QuIC products.

\section{Methods}

Brain tissue samples and compliance with ethical standards

Deidentified post-mortem brain samples were obtained from sources indicated in Table 1 and Acknowledgements in [21]. As samples were obtained from deceased, de-identified, consenting individuals, no further ethical approval was required.

\section{Neuropathology}

Brains analyzed in this work were prepared as previously described with the numbering of patients following that in Table 1 in [21]. Briefly, one half of a patient brain was formalin fixed and the other half frozen. Diagnoses of

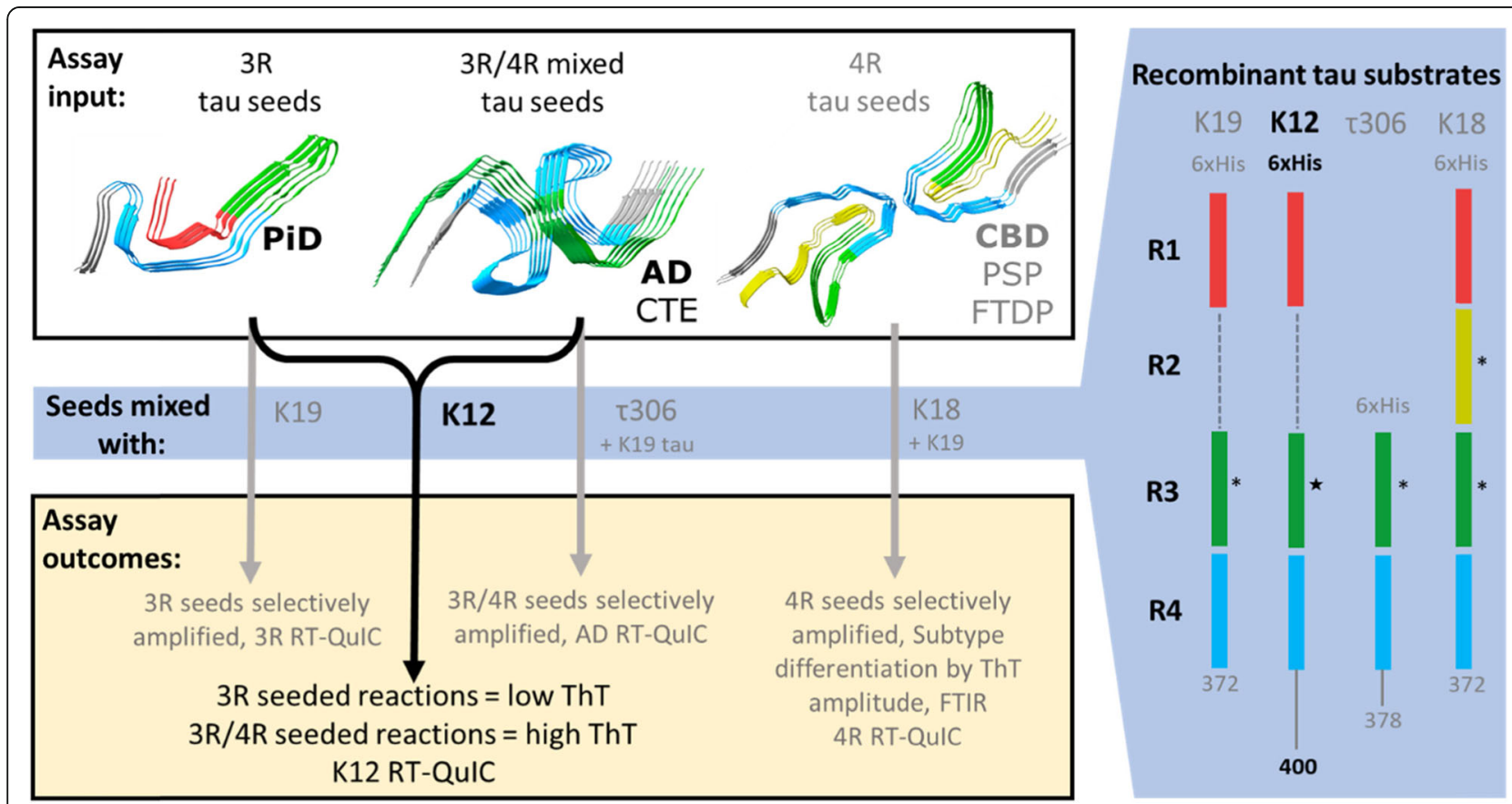

Fig. 1 Comparison of the K12 tau RT-QulC assay described in this work with the other three currently available tau RT-QulC assays. Fibril structures were taken from PDB files originally published in $[14-16,36]$. Cysteines in the canonical tau sequences were mutated to serines $\left(^{*}\right)$ or alanine $(\star)$ for recombinant tau fragments used in tau RT-QulC assays. The 3R, AD, and 4R RT-QulC assays mentioned in this figure have been described previously $[21,23,27,29]$ 
Table 1 End-point quantification of tau seeding activity in brain tissue across tau RT-QulC assays

\begin{tabular}{|c|c|c|c|c|c|c|c|c|c|}
\hline \multirow{2}{*}{$\begin{array}{l}\text { Tau aggregate } \\
\text { classification }\end{array}$} & \multirow{2}{*}{$1^{\circ}\left(2^{\circ}, 3^{\circ}, 4^{\circ}\right)$ Diagnosis } & \multirow{2}{*}{$\begin{array}{l}\text { Brain } \\
\text { region }\end{array}$} & \multirow[b]{2}{*}{ Sex } & \multirow[b]{2}{*}{ AOD } & \multirow[b]{2}{*}{ PMI (h) } & \multicolumn{4}{|c|}{ Estimated tau seed concentration (log SD 50/mg tissue) } \\
\hline & & & & & & K12 RT-QulC & AD RT QulC ${ }^{21}$ & 3R RT-QuIC ${ }^{27}$ & 4R RT-QulC ${ }^{29}$ \\
\hline \multirow{8}{*}{$3 R$} & PiD 1 (CVD) & $\mathrm{F}$ & M & 56 & 9 & $7.3 \pm 0.2$ & $5.9 \pm 0.5$ & $7.3 \pm 0.8$ & $2.6 \pm 0.1$ \\
\hline & PiD 2 ( $\beta$-amyloid pathology, CVD) & $\mathrm{F}$ & M & 64 & 3 & $8.0 \pm 0.1$ & $6.7 \pm 1.1$ & $8.3 \pm 0.4$ & $3.8 \pm 0.5$ \\
\hline & PiD 3 (CVD) & $\mathrm{F}$ & $\mathrm{F}$ & 67 & 8 & $6.5 \pm 0.2$ & & $8.1 \pm 0.8$ & $4.9 \pm 0.1$ \\
\hline & PiD 4 & $\mathrm{~F}$ & M & 70 & NA & $7.5 \pm 0.1$ & 5.0 & $7.6 \pm 0.3$ & $3.6 \pm 0.6$ \\
\hline & PiD 5 (CVD) & $\mathrm{F}$ & M & 65 & 13 & $7.3 \pm 0.3$ & & $7.7 \pm 0.2$ & $4.3 \pm 0.4$ \\
\hline & PiD 6 (CVD) & $\mathrm{F}$ & $\mathrm{F}$ & 77 & 9 & $7.3 \pm 0.3$ & & $7.2 \pm 1.2$ & $2.8 \pm 0.4$ \\
\hline & PiD 7 (SC, CVD) & $\mathrm{F}$ & $\mathrm{F}$ & 73 & 24 & $8.5 \pm 0.3$ & & $7.5 \pm 0.2$ & $3.7 \pm 1.0$ \\
\hline & PiD 8 & $\mathrm{~F}$ & M & 61 & 25 & $7.3 \pm 0.2$ & & $7.1 \pm 0.5$ & $5.2 \pm 0.3$ \\
\hline \multirow{13}{*}{$3 R / 4 R$} & SAD 1 & $\mathrm{~F}$ & M & 58 & 13 & $8.8 \pm 0.2$ & $8.8 \pm 0.1$ & $3.7 \pm 0.5$ & $2.7 \pm 0.1$ \\
\hline & SAD 2 & $\mathrm{~F}$ & $\mathrm{~F}$ & 80 & 26 & $8.0 \pm 0.4$ & $8.7 \pm 0.3$ & $3.5 \pm 0.6$ & $3.5 \pm 0.4$ \\
\hline & SAD 3 & $\mathrm{~F}$ & M & 70 & 9 & $7.8 \pm 0.2$ & $7.8 \pm 0.0$ & $3.1 \pm 0.5$ & $2.8 \pm 0.4$ \\
\hline & SAD 4 & $\mathrm{~F}$ & $\mathrm{~F}$ & 87 & 8 & $8.5 \pm 0.2$ & $8.0 \pm 1.1$ & $2.7 \pm 0.3$ & $3.6 \pm 0.5$ \\
\hline & SAD 5 & $\mathrm{~F}$ & M & 71 & 2 & $8.0 \pm 0.2$ & $8.3 \pm 0.7$ & $3.1 \pm 0.8$ & $3.6 \pm 0.3$ \\
\hline & SAD 6 & $\mathrm{~F}$ & $\mathrm{~F}$ & 84 & 33 & $8.2 \pm 0.1$ & $8.9 \pm 0.8$ & $3.0 \pm 0.3$ & $2.5 \pm 0.3$ \\
\hline & fAD-A431E 1 & $\mathrm{~F}$ & M & 44 & NA & $8.8 \pm 0.4$ & $9.5 \pm 0.4$ & 4.0 & $4.4 \pm 1.0$ \\
\hline & fAD-A431E 3 & $\mathrm{~F}$ & & & NA & $8.5 \pm 0.2$ & $9.0 \pm 0.7$ & 4.2 & $3.2 \pm 0.4$ \\
\hline & CTE 1 (DLB) & T & M & 78 & $<24$ & $7.5 \pm 0.1$ & $7.0 \pm 0.0$ & & $2.7 \pm 0.4$ \\
\hline & СTE 2 & $\mathrm{~T}$ & $M$ & 67 & $<24$ & $7.6 \pm 0.1$ & $7.5 \pm 0.4$ & & $3.0 \pm 0.5$ \\
\hline & PART 1 (LBD, CVD) & T & $\mathrm{F}$ & 77 & 4 & $6.5 \pm 0.2$ & 6.5 & & 2.5 \\
\hline & PART 2 (ALS, CVD) & $\mathrm{T}$ & M & 62 & 4 & $5.5 \pm 0.2$ & 6.0 & & 3.0 \\
\hline & PART 3 (SVD, TDP43, CVD) & T & M & 65 & 5 & $5.3 \pm 0.2$ & 5.5 & & 2.5 \\
\hline & PSP 1 & $\mathrm{~F}$ & M & 86 & 7 & $5.4 \pm 0.1$ & & $3.5 \pm 0.5$ & $6.1 \pm 1.4$ \\
\hline & PSP 2 (CVD) & $\mathrm{F}$ & M & 71 & 13 & $4.4 \pm 0.1$ & & $3.0 \pm 0.8$ & $7.0 \pm 0.4$ \\
\hline & PSP 3 (CVD) & $\mathrm{F}$ & M & 65 & 8 & $4.8 \pm 0.2$ & $5.4 \pm 0.2$ & $3.0 \pm 0.2$ & $6.6 \pm 0.1$ \\
\hline & PSP 4 (SC) & $\mathrm{F}$ & M & 67 & 4 & $5.3 \pm 0.2$ & $6.0 \pm 0.4$ & $3.9 \pm 1.0$ & $5.7 \pm 0.6$ \\
\hline & PSP 5 (CVD) & $\mathrm{F}$ & M & 76 & 6 & $5.0 \pm 0.2$ & $4.3 \pm 0.0$ & $3.6 \pm 0.9$ & $8.5 \pm 0.8$ \\
\hline & PSP 6 & $\mathrm{~F}$ & $\mathrm{~F}$ & 71 & 7 & $3.5 \pm 0.2$ & & $2.7 \pm 0.5$ & $5.9 \pm 0.7$ \\
\hline & CBD 1 & $\mathrm{~F}$ & $\mathrm{~F}$ & 51 & 10 & $5.4 \pm 0.2$ & $5.8 \pm 0.2$ & $2.5 \pm 0.3$ & $7.2 \pm 0.7$ \\
\hline $4 \mathrm{R}$ & CBD 2 (CVD, SC) & $\mathrm{F}$ & $\mathrm{F}$ & 75 & 51 & $6.3 \pm 0.2$ & & $2.9 \pm 0.1$ & $7.1 \pm 0.7$ \\
\hline & CBD 3 (CVD) & $\mathrm{F}$ & M & 65 & 17 & $5.6 \pm 0.1$ & $6.6 \pm 0.2$ & $3.2 \pm 0.6$ & $8.2 \pm 0.5$ \\
\hline & CBD 4 (CVD, SC) & $\mathrm{F}$ & $F$ & 69 & 5 & $4.9 \pm 0.2$ & & $2.5 \pm 0.3$ & $7.6 \pm 0.5$ \\
\hline & AGD 2 (CVD) & $\mathrm{F}$ & M & 91 & 2 & $5.9 \pm 0.2$ & 5.5 & $3.6 \pm 0.4$ & \\
\hline & AGD 3 (HS) & $\mathrm{F}$ & M & 86 & 110 & $4.8 \pm 0.2$ & & $3.6 \pm 0.4$ & \\
\hline & FTDP-17 3 & $\mathrm{~F}$ & $\mathrm{~F}$ & 54 & 11 & $5.3 \pm 0.2$ & $5.7 \pm 0.8$ & $3.1 \pm 0.1$ & $6.9 \pm 0.5$ \\
\hline & MSA 1 & $\mathrm{~F}$ & $\mathrm{~F}$ & 71 & NA & $5.0 \pm 0.2$ & & & $2.3 \pm 0.2$ \\
\hline & MSA 2 & $\mathrm{~F}$ & M & 52 & 3 & $4.0 \pm 0.2$ & & & $3.3 \pm 0.2$ \\
\hline & PD 2 & $\mathrm{~F}$ & M & 61 & $U$ & $3.1 \pm 0.2$ & $5.1 \pm 0.7$ & & \\
\hline & DLBD 2 (SC, CM, CVD) & $\mathrm{F}$ & $M$ & 81 & 20 & $4.6 \pm 0.1$ & & $2.3 \pm 0.2$ & $2.4 \pm 0.2$ \\
\hline & DLBD 3 (SC, CVD) & $\mathrm{F}$ & M & 73 & 14 & $4.3 \pm 0.2$ & & $2.6 \pm 0.6$ & $2.6 \pm 0.5$ \\
\hline & DLBD 4 (CVD, SC) & $\mathrm{F}$ & M & 71 & 8 & $5.5 \pm 0.1$ & 5.3 & $2.4 \pm 0.2$ & $2.9 \pm 0.4$ \\
\hline & FTLD-TDP 1 (CVD) & $\mathrm{F}$ & $\mathrm{F}$ & 50 & 6 & $5.0 \pm 0.1$ & 5.3 & $4.2 \pm 0.4$ & $2.8 \pm 0.4$ \\
\hline & FTLD-TDP-Type C 3 (CVD) & $\mathrm{F}$ & $\mathrm{F}$ & 68 & 15 & $4.6 \pm 0.1$ & & $2.9 \pm 0.9$ & 2.5 \\
\hline No/low & FTLD-TDP-Type A 4 (CVD) & $\mathrm{F}$ & $\mathrm{F}$ & 65 & 5 & $3.5 \pm 0.3$ & $4.0 \pm 1.0$ & $3.6 \pm 0.1$ & 2.5 \\
\hline & SC 1 & $\mathrm{~F}$ & $\mathrm{~F}$ & 76 & 4 & $5.4 \pm 0.0$ & $5.3 \pm 0.4$ & $2.8 \pm 0.4$ & $2.6 \pm 0.1$ \\
\hline & SC 2 (Meningioma) & $\mathrm{F}$ & $\mathrm{F}$ & 73 & 24 & $5.3 \pm 0.2$ & & $3.2 \pm 0.6$ & $3.1 \pm 0.3$ \\
\hline & $\mathrm{SC} 3$ & $\mathrm{~F}$ & $\mathrm{~F}$ & 81 & 25 & $4.6 \pm 0.2$ & $5.3 \pm 0.6$ & $2.7 \pm 0.5$ & $2.6 \pm 0.1$ \\
\hline & CVD 1 & $\mathrm{~F}$ & $\mathrm{~F}$ & 53 & 19 & $3.3 \pm 0.2$ & $4.3 \pm 0.4$ & $2.4 \pm 0.2$ & 2.2 \\
\hline & CVD 2 & $\mathrm{~F}$ & $\mathrm{~F}$ & 66 & 17 & $4.9 \pm 0.2$ & & $2.8 \pm 0.6$ & $2.4 \pm 0.2$ \\
\hline & CVD 3 (SC) & $\mathrm{F}$ & $\mathrm{F}$ & 77 & 6 & $4.4 \pm 0.1$ & $3.7 \pm 0.8$ & $3.0 \pm 0.3$ & $3.0 \pm 0.7$ \\
\hline & CVD 4 (SAH, SC) & $\mathrm{F}$ & $\mathrm{F}$ & 86 & NA & $4.6 \pm 0.2$ & $4.4 \pm 1.7$ & $3.2 \pm 0.1$ & $3.7 \pm 1.0$ \\
\hline & ALS 1 (SC, CVD) & $\mathrm{F}$ & M & 72 & 2 & $4.8 \pm 0.2$ & $4.9 \pm 0.2$ & $2.6 \pm 0.1$ & $2.4 \pm 0.2$ \\
\hline tau KO & & & & & & 2.3 & 2.5 & 2.3 & $2.5 \pm 0.4$ \\
\hline Brain region: & $\mathrm{F}$, frontal cortex & & & & HS & Hippocampal sc & clerosis & & \\
\hline & $\mathrm{T}$, temporal cortex & & & & $\mathrm{NH}$ & Intraventricular h & hemorrhage & & \\
\hline & & & & & MSA & Multiple system & atrophy & & \\
\hline AGD & Argyrophilic grain disease & & & & NA & Not available & & & \\
\hline sAD & sporadic Alzheimer disease & & & & PART & Primary age-rela & lated tauopathy & & \\
\hline fAD & familial Alzheimer disease & & & & PD & Parkinson's dise & ease & & \\
\hline ALS & Amyotrophic lateral sclerosis & & & & PiD & Pick disease & & & \\
\hline AOD & Age of death & & & & PMI & Post-mortem int & terval & & \\
\hline CTE & chronic traumatic encephalopathy & & & & PSP & Progressive sup & pranuclear palsy & & \\
\hline CBD & Corticobasal degeneration & & & & SAH & Sub-arachnoid $\mathrm{h}$ & hemorrhage & & \\
\hline CM & Cerebral malformation & & & & SC & Senile change & & & \\
\hline CVD & Cerebrovascular disease & & & & SVD & Small vessel dis & sease & & \\
\hline DLBD & Diffuse Lewy body disease & & & & tau KO & Human and mou & use tau knockout & at mouse & \\
\hline FTDP-17 & $\begin{array}{l}\text { Frontotemporal dementia with } \\
\text { parkinsonism linked to chromosome }\end{array}$ & & & & & & & & \\
\hline & 17 & & & & $\mathrm{u}$ & Information unav & vailable & & \\
\hline FTLD-TDP & Frontotemporal lobar degeneration wi & TDP-43 & & & & & & & \\
\hline
\end{tabular}


fixed tissues were made using the stains described in [6, 21]. Tissue samples in this study were collected from frontal cortex except for CTE and primary age-related tauopathy (PART) samples which were from temporal cortex. $10 \% \mathrm{w} / \mathrm{v}$ brain homogenates of frontal cortex tissue were prepared in ice-cold PBS using $1 \mathrm{~mm}$ silica beads (BioSpec, 11079110z) and Beadbeater (BioSpec) or BeadMill 24 (Fischer). K. N. provided CTE; B. G. provided all other samples, $\mathrm{PiD}, \mathrm{AD}$, diseases with $4 \mathrm{R}$ tau deposits, and controls lacking tau pathology.

\section{Purification of recombinant $\mathrm{K} 12 \mathrm{CFh}$ tau}

A cloning cassette encoding the K12 tau fragment (residues 244-275 and 306-400 of the full-length human tau sequence) with an alanine mutation at residue 322 [17], thereby rendering it cysteine-free (K12CF), was introduced between the Ndel and XhoI sites of the pET28 bacterial vector resulting in a histidine tag at the $\mathrm{N}$-terminal (K12CFh). The cysteine was mutated in order to prevent disulfide-mediated dimerization of the $\mathrm{K} 12$ molecules. Purification of K12CFh follows the steps described in [27, 28] and is summarized schematically in Additional file 1. Briefly, K12CFh was expressed in BL21(DE3) E. coli following the overnight autoinduction protocol described in [27]. Cells were centrifuged at $3750 \mathrm{rpm}$ for $35 \mathrm{~min}$ at $4{ }^{\circ} \mathrm{C}$, resuspended in buffer A $(10 \mathrm{mM}$ Tris, $500 \mathrm{mM} \mathrm{NaCl}$, $5 \mathrm{mM}$ imidazole, $\mathrm{pH} 8.0$ ), and probe sonicated $3 \times 45 \mathrm{~s}$ at $25 \%$ pulse with $15 \mathrm{~s}$ pause. Lysates were filtered through $0.45 \mu \mathrm{M}$ syringe filter prior to nickel affinity chromatography using a His-Trap FF (GE Healthcare 17-5255-01) column. Sequential wash steps with 13 and $21 \%$ buffer B (10 mM Tris, $500 \mathrm{mM} \mathrm{NaCl}, 200 \mathrm{mM}$ imidazole, $\mathrm{pH} 8.0$ ) over 5 and 7 column volumes each eluted two contaminants observed previously in K18CFh, K19CFh, and t306$378 \mathrm{CFh}$ purifications. K12CFh was eluted over a linear gradient of 46-200 mM imidazole over 8 column volumes. Fractions were pooled and precipitated in $4 \mathrm{x}$ volumes of ice-cold acetone in $4{ }^{\circ} \mathrm{C}$ overnight. Acetone precipitant was pelleted at $10,000 \times \mathrm{g}$ for $20 \mathrm{~min}$ and washed with a further $20 \mathrm{~mL}$ acetone with a repeat centrifugation at 10 , $000 \times g$ for $20 \mathrm{~min}$. Acetone was decanted and pellets dissolved in $8 \mathrm{M}$ Guanidine- $\mathrm{HCl}(\mathrm{GdnHCl})$ in PBS. The dissolved K12CFh was desalted into PBS, pH 7.0 using PD-10 desalting columns (GE Healthcare, 17-0851-01). Removal of guanidine was verified via UV absorbance readings. The $\mathrm{K} 12 \mathrm{CFh}$ concentration was adjusted to $\sim 0.75 \mathrm{mg} / \mathrm{mL}$ and aliquots stored at $-70^{\circ} \mathrm{C}$ until use.

\section{K12 RT-QulC}

The K12 RT-QuIC assay follows a modified version of the protocols described in $[27,28]$. K12CFh was thawed from $-70^{\circ} \mathrm{C}$ and filtered through $100 \mathrm{kDa}$ filters (Pall) to remove preformed aggregates. Concentration was adjusted to $6.5 \mu \mathrm{M} \sim 0.1 \mathrm{mg} / \mathrm{mL} \mathrm{K} 12 \mathrm{CFh}$ in a buffer containing $40 \mathrm{mM}$ HEPES, pH 7.4, $400 \mathrm{mM} \mathrm{NaF}, 40 \mu \mathrm{M}$ heparin, and $10 \mu \mathrm{M}$ thioflavin $\mathrm{T}(\mathrm{ThT})$. These reaction conditions were reached following the framework described in [23]. The K12CFh solution was thoroughly mixed in a polypropylene boat by gentle rocking for $10 \mathrm{~s}$ and $48 \mu \mathrm{L}$ or $49 \mu \mathrm{L}$ mix was added to each well of a 384-well optically clear bottom plate (NUNC) using a multichannel pipettor. Brain homogenates (BHs) were thawed from storage at $10 \% \mathrm{w} / \mathrm{v}$ in ice cold PBS and serially diluted in 10-fold steps using a dilution buffer containing $0.53 \%$ tau-free mouse (KO; B6.129S4(Cg)Mapt $^{\text {tm1(EGFP)Klt } / J ~ f r o m ~ J a c k s o n ~ L a b o r a t o r i e s) ~ a n d ~ 1 x ~ N-~}$ 2 Supplement (Gibco) $+10 \mathrm{mM}$ HEPES. Inclusion of tau-free mouse $\mathrm{BH}$ and $\mathrm{N}-2$ supplement were critical for preventing spontaneous aggregation of the K12CFh tau fragment. One or two $\mu \mathrm{L}$ of $\mathrm{BH}$ dilutions were seeded into quadruplicate or octuplicate wells for a final reaction volume of $50 \mu \mathrm{L}$ in each well. Plates were sealed with clear adhesive sealing tape and placed in an Omega FLUOStar plate reader pre-warmed to $42^{\circ} \mathrm{C}$ and subjected to rounds of $1 \mathrm{~min}$ shaking, $500 \mathrm{rpm}$, orbital, and 1 min rest, with ThT fluorescence reads (450 excitation, 480 emission) taken every $15 \mathrm{~min}$.

\section{FTIR analysis of K12 RT-QulC products}

Once ThT fluorescence reached a plateau, and prior to spontaneous fibril formation in mock-seeded control (tau KO-seeded) wells, reactions were stopped and RT-QuIC products were recovered by scraping the bottom of the microplate wells with a pipette tip. Eight replicate reactions seeded with a $10^{-4}$ dilution of the designated $\mathrm{BH}$ were pooled in $0.5 \mathrm{~mL}$ microfuge tubes and centrifuged at $13,100 \times \mathrm{g}$ for $10 \mathrm{~min}$, at $4{ }^{\circ} \mathrm{C}$. Supernatant containing soluble $\mathrm{K} 12 \mathrm{CFh}$ and other buffer components was discarded, and pellets were washed twice with $400 \mu \mathrm{L} \mathrm{H}_{2} \mathrm{O}$. Pellets were resuspended in $5 \mu \mathrm{L} \mathrm{H}_{2} \mathrm{O}$ and subjected to attenuated total reflectance (ATR) Fourier transform infrared (FTIR) spectroscopy. $1.5 \mu \mathrm{L} \mathrm{H}_{2} \mathrm{O}$-protein slurry was applied to a Perkin-Elmer Spectrum100 FTIR with an ATR diamond attachment and dried with a gentle flow of dry air. Prior to scanning, sample and electronics chambers were purged with a constant flow of dry air. 100 replicate scans were averaged from 4000 to $800 \mathrm{~cm}^{-1}$, normalized to amide I intensity ( $\sim 1630 \mathrm{~cm}^{-1}$ peak), and second derivatives were taken with 9 points for slope analysis.

\section{Electron microscopy of K12 RT-QulC products}

Prior to pelleting RT-QuIC products for FTIR, $\sim 50 \mu \mathrm{L}$ of pooled reactions seeded with $10^{-4} \mathrm{BH}$ dilutions were taken and wicked onto grids for transmission electron microscopy (TEM). Ultrathin C film on lacey carbon support film (400 mesh, Copper, TedPella Prod\# 01824) were glow discharged and incubated with fibril solutions for $30 \mathrm{~min}$ at room temperature. Grids were washed 3 
times with Milli-Q (Millipore)-purified water prior to being stained with Nano-W (Nanoprobes \#2018-5) stain for $15 \mathrm{~min}$ and wicked dry. Images were acquired on a T12 (Thermo Fisher) transmission electron microscope operating at $120 \mathrm{kV}$ with a Rio (Gatan) CMOS camera.

\section{LogS $\mathrm{S}_{50}$ and standard error statistical calculation}

As a measure of seed concentrations in samples, we employed $\log \mathrm{SD}_{50}$ calculations using the Spearman-Kärber method, which determine the $\log _{10}$ dilution at which $50 \%$ of wells give ThT fluorescence greater than 100 times the standard deviation of baseline fluorescence (threshold), prior to $60 \mathrm{~h}$ [11]. $\mathrm{LogSD}_{50} / \mathrm{mg}$ tissue values represent a determination from a single serial dilution of a brain homogenate with eight replicate reactions at dilutions from $10^{-3}-10^{-10}$, with a final adjustment to report $\mathrm{SD}_{50}$ seeding units per mg brain tissue. $\operatorname{LogSD_{50}}=x_{p=1}+1 / 2 d-d \sum p$ with $x_{p=1}$ being the highest $\log$ dilution giving $8 / 8$ positive responses; $d=\log$ dilution factor; $p=$ proportion positive at a given dose; $\Sigma p=$ the sum of values of $p$ for $x_{p}=1$ and all higher dilutions [11]. Error bars represent the standard error calculated with the formula $[\Sigma(p(1-p)) / \mathrm{n}-1)]^{1 / 2}$ where $\mathrm{n}=$ number of replicates. This method of error estimation is further described in [11].

\section{Analytical sensitivity estimation}

To estimate the analytical sensitivity of K12 tau RT-QuIC, synthetic K12CFh fibrils were assayed over serial dilutions of a known concentration of starting fibrils. Fibrils were generated by seeding $6.5 \mu \mathrm{M}$ K12CFh substrate with either $\mathrm{AD}$, $\mathrm{PiD}$, or $\mathrm{KO} \mathrm{BH}$ at $10^{-4}$ dilution in conditions identical to those described above for K12 RT-QuIC. Fibrils were scraped from the microwell plate and analyzed by SDS-PAGE for percent conversion of substrate to approximate the concentration of fibrils generated. We estimated that nearly $\sim 100 \%$ of the K12CFh was converted in all reaction conditions. Serial dilutions of fibrils was conducted identically to those for brain homogenate, in a background of $10 \mathrm{mM}$ HEPES $\mathrm{pH} 7.4,1 \mathrm{x} \mathrm{N}-2$, and $0.53 \% \mathrm{KO} \mathrm{BH}$.

\section{Results}

$\mathrm{K} 12 \mathrm{CFh}$ fibrillizes in the presence of $3 \mathrm{R}$ and $3 \mathrm{R} / 4 \mathrm{R}$ tau seeds Multiple reaction conditions including temperature, shaking speed, substrate concentration, addition of salts, and co-factors were tested before arriving at the present $\mathrm{K} 12$ RT-QuIC reaction conditions. Using a strategy we described previously [23], we found that $400 \mathrm{mM} \mathrm{NaF}$ provided the highest fold separation of lag phases (time to threshold at which ThT fluorescence exceeded 100 standard deviations of the baseline fluorescence) between positive (AD- and PiD-seeded) and negative (KO-seeded) reactions (Additional file 2). Using these reaction conditions, we observed seeded fibrillization of the K12CFh substrate, indicated by increased ThT fluorescence, in some reactions seeded with brain homogenate dilutions as extreme as $10^{-9}$ and $10^{-8}$ for $\mathrm{AD}$ and $\mathrm{PiD}$, respectively,

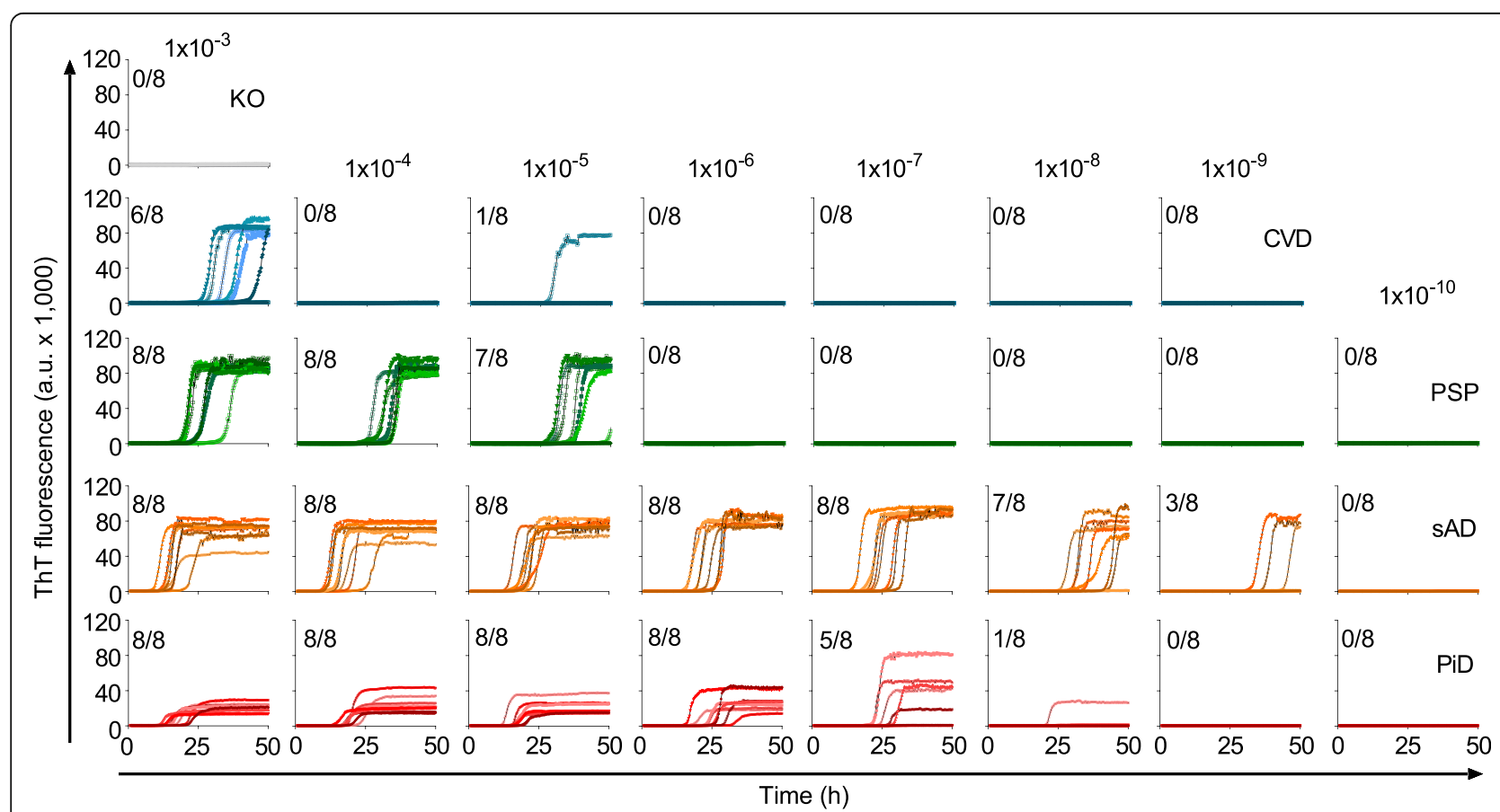

Fig. 2 Primary ThT fluorescence data of K12 RT-QulC endpoint dilution analyses. Panels show traces from 8 replicate reactions at the designated dilutions of tau-free mouse brain (KO), cerebrovascular disease (CVD), progressive supranuclear palsy (PSP), Alzheimer disease (AD), and Pick disease (PiD) brain homogenates. The fractions in the upper left corner of each panel indicate the ThT-positive/total replicate reactions 
within $50 \mathrm{~h}$ (Fig. 2). In contrast, brains that either lacked immunohistochemically detectable tau pathology, e.g. a cerebrovascular disease (CVD) case, or had 4R tau pathology (a PSP case), seeded fibrillization of $\mathrm{K} 12 \mathrm{CFh}$ at only $10^{-3}-10^{-5}$ dilution of brain homogenate. These initial experiments indicated preference of the $\mathrm{K} 12 \mathrm{CFh}$ substrate for seeding by the tau aggregates of $\mathrm{AD}$ and $\mathrm{PiD}$.

\section{K12CFh detects PiD, AD, CTE seeds preferentially over $4 R$} tau seeds

To further explore the seeding selectivity of the K12CFh substrate, we performed serial dilution analyses on several brain homogenates with confirmed 3R pathology (PiD), mixed 3R/4R pathology (sAD, fAD, CTE, PART), and controls that had either 4R pathology (PSP, CBD, AGD) or lacked tau pathology according to immunohistochemical analysis. Figure 3 summarizes these data in terms of $\log \mathrm{SD}_{50} / \mathrm{mg}$ tissue. An $\mathrm{SD}_{50}$ is a unit of seeding activity that gives a positive reaction in $50 \%$ of replicate reactions. Thus $\log \mathrm{SD}_{50} / \mathrm{mg}$ tissue values represent the concentration of seeding units measured by the assay. $\mathrm{PiD} \mathrm{BHs}$ had a mean $\log \mathrm{SD}_{50} / \mathrm{mg}$ tissue of $7.5 \pm 0.6$ ( \pm standard deviation), very similar to that obtained previously using the 3R RT-QuIC assay [27]. The AD and CTE brains with 3R/ $4 \mathrm{R}$ tau pathology had similarly high tau seeding activities of 8.3 $\pm 0.4(n=8)$ and 7.5-7.6 $(n=2)$, respectively. The brains with $4 \mathrm{R}$ tau pathology reactions all had much lower seeding activities (mean $\log \mathrm{SD}_{50} / \mathrm{mg}$ tissue $=5.1 \pm 0.7$; $n=13$ ), albeit higher than those of brains that were immunohistochemically negative for tau pathology (mean log

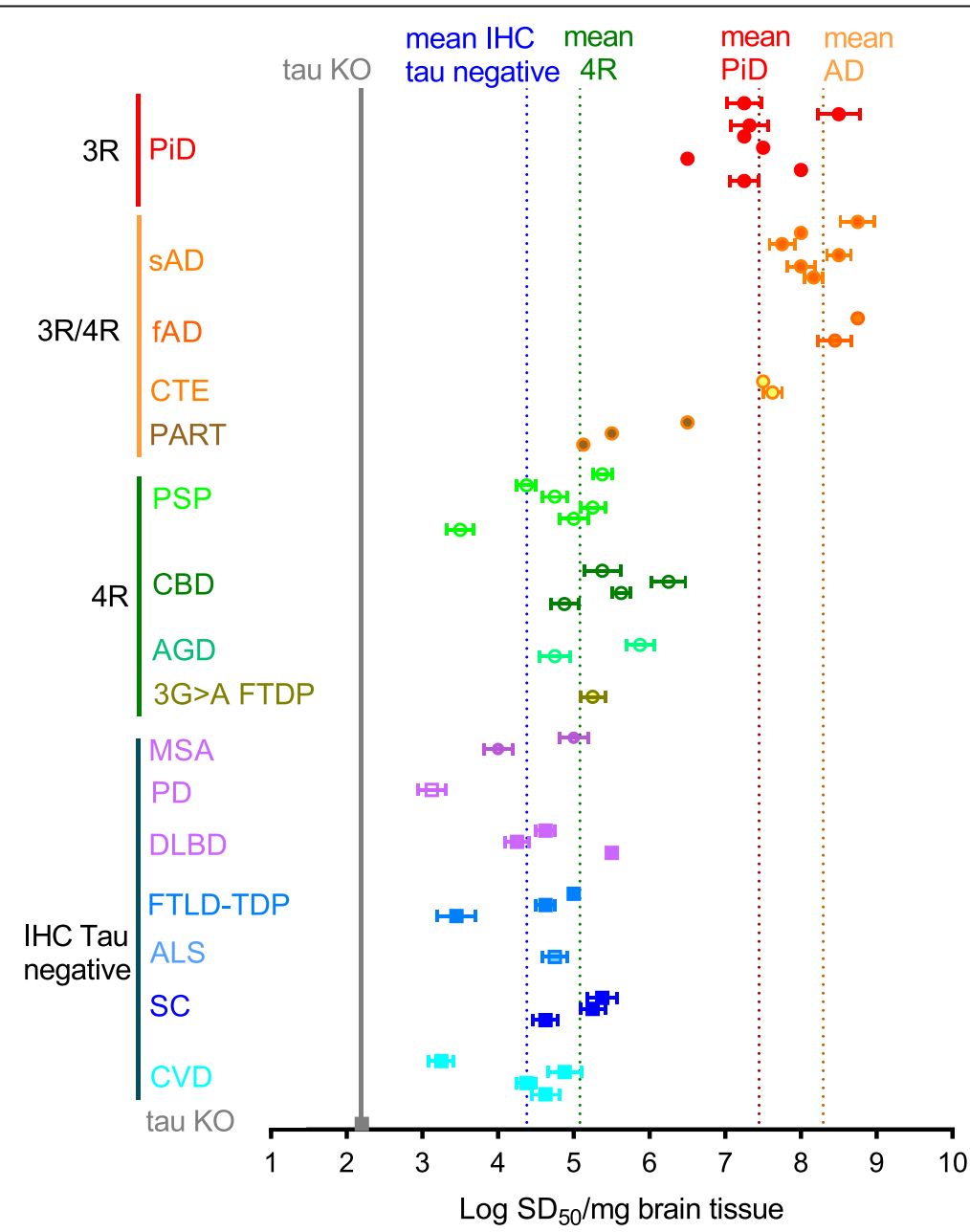

Fig. 3 Seed concentration (LogSD 50 /mg brain) determinations of 3R, 3R/4R, 4R, and immunohistochemically (IHC) tau-negative brain homogenates by K12 RT-QulC. Points represent $\operatorname{logSD}_{50} / \mathrm{mg}$ brain values calculated for a dilution series with a brain homogenate with 8 replicate reactions at each dilution. Error bars represent the standard error of $\operatorname{logSD}_{50} / \mathrm{mg}$ brain determined by the equation described in Methods. Vertical dotted lines represent mean logSD $/$ /mg brain values across the designated brain homogenate types. PiD, Pick disease; SAD, sporadic AD; fAD, familial AD; CTE, chronic traumatic encephalopathy; PART, primary age-related tauopathy; PSP, progressive supranuclear palsy; CBD, corticobasal degeneration; AGD, argyrophilic grain disease; IVS10 + 3G > A FTDP, frontotemporal dementia with parkinsonism; MSA, multiple system atrophy; PD, Parkinson's disease; DLBD, diffuse Lewy body dementia; FTLD-TDP43, frontotemporal lobar degeneration with TDP43 protein; ALS, amyotrophic lateral sclerosis; SC, senile change; CVD, cerebrovascular disease 
$\mathrm{SD}_{50} / \mathrm{mg}$ tissue $=4.5 \pm 0.7 ; n=17$ ). Cut-off times for log $\mathrm{SD}_{50} / \mathrm{mg}$ tissue determinations were set to $60 \mathrm{~h}$ based on observations that no further PiD-, AD-, or CTE-seeded reactions exceeded 100 standard deviations of the baseline ThT fluorescence at time points beyond $60 \mathrm{~h}$. Spontaneous conversion of $\mathrm{K} 12 \mathrm{CFh}$ was not observed in tau KOseeded wells prior to $120 \mathrm{~h}$. Spearman-Kärber based estimations of $\log \mathrm{SD}_{50} / \mathrm{mg}$ tissue values for various tau aggregation disease cases and controls are summarized in
Table 1 for comparison between K12, AD (3R/4R), 3R, and 4R tau RT-QuIC assays.

\section{Maximum ThT fluorescence distinguishes 3R- from 3R/4R- seeded reactions}

Our initial comparisons of AD- and PiD-seeded K12 RTQuIC reactions indicated several fold higher maximum ThT fluorescence amplitudes in the AD-seeded reactions (Fig. 2, bottom two rows). Remarkably, this amplitude

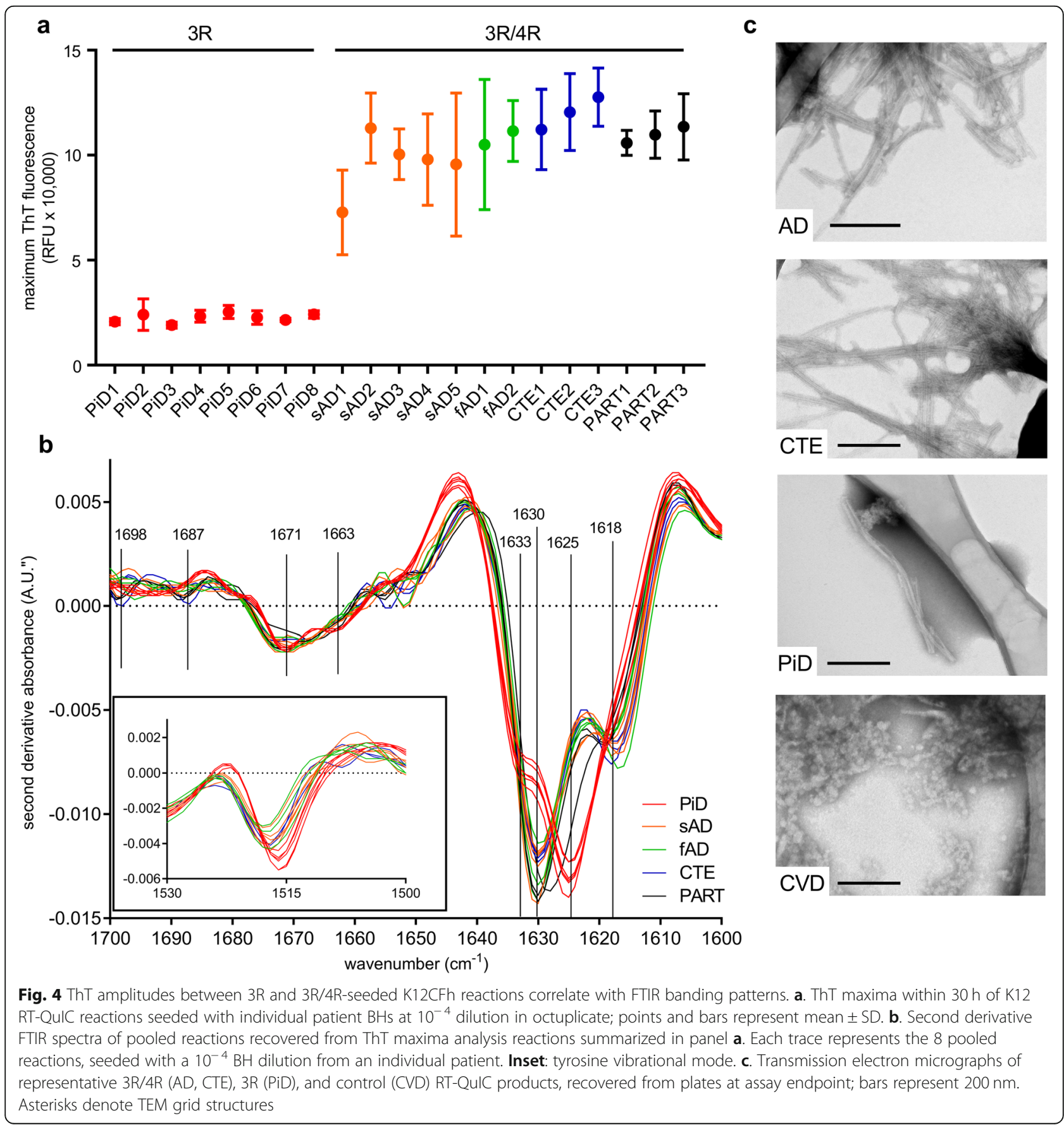


difference was seen over a large $\left(10^{-3}-10^{-6}\right)$ range of $\mathrm{BH}$ dilutions indicating that it was primarily the character, rather than the concentration, of the seeds in the AD versus $\mathrm{PiD}$ brain specimens that determined the divergent fluorescence amplitudes. These fluorescence maxima differences were also seen in comparisons of reactions seeded with $8 \mathrm{PiD}$ brain homogenates $(\sim 21,000$ relative fluorescence units) compared to $2 \mathrm{fAD}, 6 \mathrm{sAD}, 2 \mathrm{CTE}$, and 3 PART brain homogenates $(\sim 50,000-150,000)$ (Fig. 4a). Modifying reaction conditions such as temperature, beads, fluorescence gain setting, or concentrations of $\mathrm{K} 12 \mathrm{CFh}$, ThT, NaF, and heparin all changed absolute maximum ThT values, but relative amplitude differences between $\mathrm{PiD}$ - and $\mathrm{AD}$-seeded reactions remained (Additional file 3). These results indicated that $\mathrm{PiD}$ and $\mathrm{AD}$ seeds induced the formation of distinct K12 RT-QuIC products over a range of conditions. However, at extreme (near end-point) dilutions of $\mathrm{PiD} \mathrm{BH}$, the consistency of the relatively low ThT maxima was diminished, as observed with a $10^{-7}$ dilution in Fig. 2.

\section{ThT fluorescence amplitudes correlate with distinct fibril structures of 3R versus 3R/4R-seeded K12 RT-QulC reactions}

To investigate if ThT fluorescence differences correlate to structural properties of the brain homogenate-seeded fibrils, we recovered RT-QuIC products from microwell plates and analyzed them by ATR-FTIR spectroscopy. Figure $4 \mathrm{~b}$ shows second derivative FTIR spectra of 3Rseeded (red traces) and 3R/4R-seeded (green, orange, blue

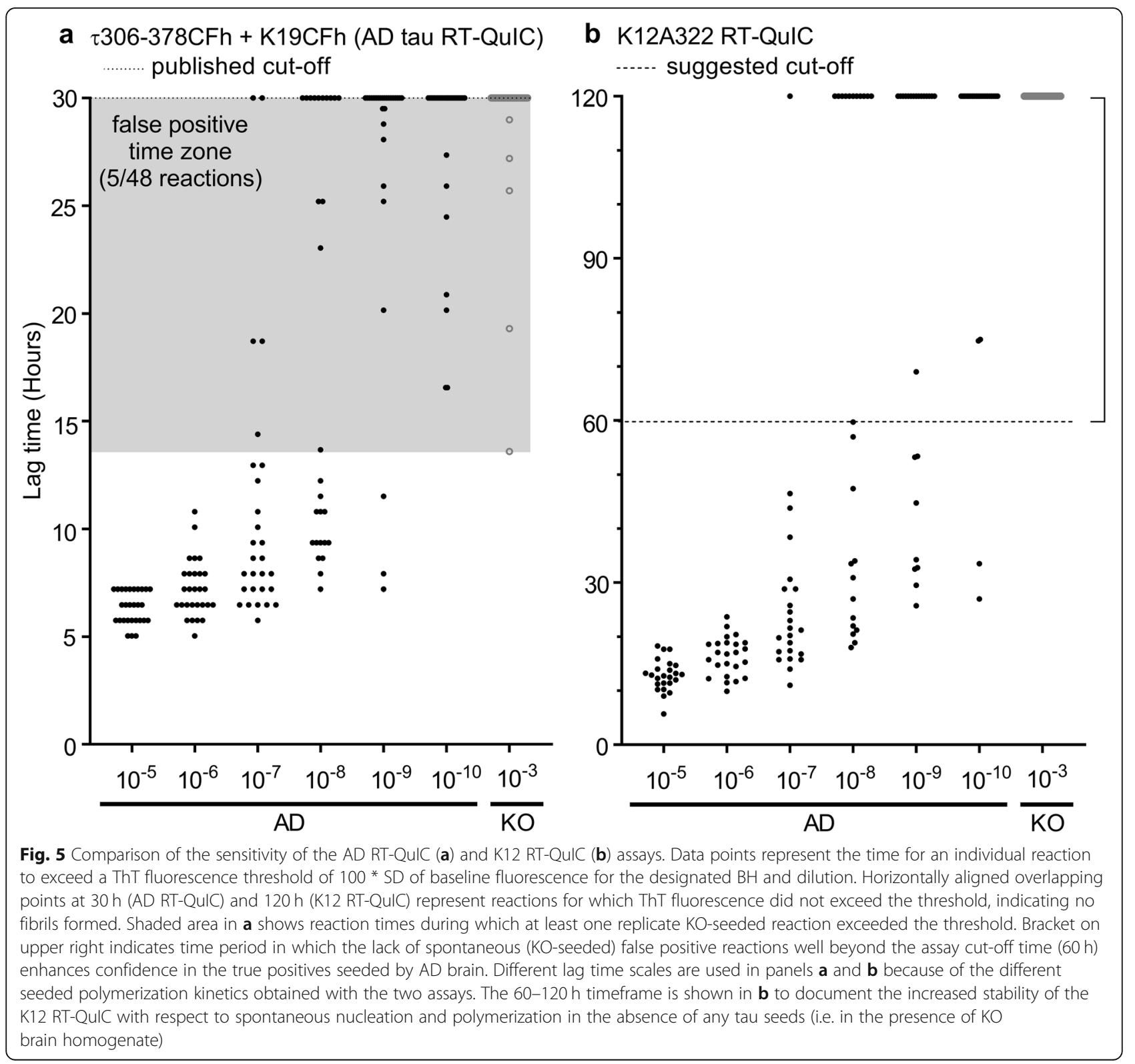


traces) K12 tau RT-QuIC products. All traces suggest high $\beta$-sheet content in the reaction products with several peaks in the $\beta$-sheet region $\left(1633,1630,1625,1618 \mathrm{~cm}^{-1}\right)$. The spectra of the PiD-seeded reaction products had prominent $1633,1625 \mathrm{~cm}^{-1} \beta$-sheet vibrational modes, which were distinct from $3 \mathrm{R} / 4 \mathrm{R}$-seeded reaction products with prominent 1630 and $1618 \mathrm{~cm}^{-1}$ vibrations; these results provide evidence that two distinct conformers of K12CFh aggregates resulted from the differential seeding (3R versus 3R/4R) of K12 RT-QuIC reactions. Further spectral differences were observed in the $1655-1700 \mathrm{~cm}^{-1}$ region containing turn and other parallel/antiparallel $\beta$-sheet bands as well as at $1516-7 \mathrm{~cm}^{-1}$ where tyrosine absorbs (Fig. 4b inset). Even further differences were evident in the fingerprint region below $1400 \mathrm{~cm}^{-1}$, which is rich in side chain vibrations (Additional file 4).

We confirmed the fibrillar morphology of K12 RTQuIC reaction products with transmission electron microscopy which indicated abundant elongated fibrils in the AD-, PiD-, and CTE-seeded, but not in control (CVD)-seeded, K12CFh products (Fig. 4c).

\section{Mixed AD/PiD pathology experiments}

To investigate what might be observed when assaying samples with mixed $\mathrm{AD} / \mathrm{PiD}$ pathology, we took $\mathrm{AD}$ and PiD brain homogenates with nominally comparable $\log \mathrm{SD}_{50} / \mathrm{mg}$ tissue values, thus representing similar (within several fold) seeding doses of the tau aggregates therein, and mixed them together in ratios ranging from 10:0-0:10 AD:PiD before analyzing ThT fluorescence amplitudes in the K12 RT-QuIC. Additional file 5 summarizes the maximum ThT values observed over decreasing $\mathrm{AD}: \mathrm{PiD}$ ratio at a total $\mathrm{BH}$ dilution of $10^{-4}$. Reactions seeded only with AD BH (10:0 AD:PiD) showed characteristically high ThT fluorescence values that decrease with a decreasing ratio of AD:PiD. However, surprisingly, even with the addition of $1 / 10$ equivalent of $\mathrm{PiD} \mathrm{BH}$, the ThT fluorescence maxima of the reactions dropped to levels approaching those obtained with PiD BH alone. These results suggested that either (1) PiD tau seeds are more efficient at seeding K12CFh fibrillization, or (2) that this PiD BH had a several fold higher seed concentration than the $\mathrm{AD} B \mathrm{BH}$, which is possible because of $\sim 0.5 \log$ margin of error that we have observed is typical of end-point dilution RT-QuIC titrations.

\section{K12 RT-QulC is as sensitive as AD RT-QulC}

By performing K12 RT-QuIC analysis on serial dilutions of synthetic K12CFh fibrils, we were able to crudely estimate the concentration of fibrils that the K12 RT-QuIC assay can detect. Additional file 6 shows lag time analysis of serial dilutions of synthetic $\mathrm{K} 12 \mathrm{CFh}$ fibrils generated by seeding with tau $\mathrm{KO}$ mouse $\mathrm{BH}$ (left), $\mathrm{AD} \mathrm{BH}$ (middle), and $\mathrm{PiD} \mathrm{BH}$ (right) at known fibril concentrations in $\mathrm{K} 12$ RT-QuIC. Individual points represent the time to threshold ThT fluorescence (fluorescence $>100 *$ SD baseline) for 8 replicate reactions at the indicated dilutions. By Spearman-Kärber analysis, the analytical sensitivity of the assay was roughly $\sim 75 \mathrm{fM}$ for synthetic AD-seeded K12CFh fibrils, and $7.5 \mathrm{fM}$ for both synthetic PiDseeded K12CFh fibrils and spontaneously converted (tau KO mouse-seeded) fibrils, comparable to the analytical sensitivity of the previously published AD tau RT-QuIC [21]. Finally, we compared the AD and K12 RT-QuIC assays by endpoint analysis of 7 AD BHs. Figure 5 shows the lag time, defined above, for $7 \mathrm{AD}$ BHs assayed in AD RTQuIC (5A) versus in K12 RT-QuIC (5B). Grey circles in both panels represent $\mathrm{KO} \mathrm{BH}$-seeded reactions, which serve as negative controls. The AD RT-QuIC showed occasional $(5 / 48)$ positive reactions occurring in $\mathrm{KO}$-seeded wells, prior to the published assay endpoint of $30 \mathrm{~h}$ (grey shaded area). Nonetheless, similar mean seed concentrations for AD brain samples were obtained with SpearmanKärber analyses of end-point dilution data the two assays, i.e., $8.7+/-0.2$ and $8.4+/-0.2 \log \mathrm{SD}_{50} / \mathrm{mg}$ with $\mathrm{AD}$ tau RT-QuIC and K12 RT-QuIC, respectively.

\section{Discussion}

Given that tau deposition appears to underlie the pathogenesis of multiple neurodegenerative diseases, it is important to have robust and practical assays for different types of tau aggregates for use in diagnostics and research. Tau RTQuIC assays exploit the fundamental self-propagating activity of pathological forms of tau, offering detection methods that are more sensitive than, and complementary to, conventional methods such as immunohistochemistry, immunoblotting, and ELISA. Thus, tau RT-QuIC assays allow measurements of multiple types of tau aggregates whether they represent the predominant cause of neurodegeneration or much less abundant co-pathology that is secondary to another disease process. Our K12 RT-QuIC assay allows the ultrasensitive detection and discrimination of both $3 \mathrm{R}$ and $3 R / 4 R$ types of pathological tau using a single tau substrate (K12CFh). From a practical perspective, this assay halves the number of assays and recombinant tau substrates required to measure these types of tau seeds. More fundamentally, our findings also demonstrate the different templating activities of $3 \mathrm{R}$ versus $3 \mathrm{R} / 4 \mathrm{R}$ tau-associated aggregates. However, our observation that the discriminatory ability of the assay based on maximum ThT fluorescence can break down with extreme near-end-point dilutions of BH (Fig. 2) suggests that 3R vs 3R/4R discrimination should be performed on specimens with seed concentrations that are at least 10-fold above the end-point dilution of the assay. Based on our current observations, K12 RT-QuIC could be applied to postmortem brain samples to aid in the characterization of decedents' molecular 
pathologies for diagnostic and research purposes. Presumably, it can be similarly applied in animal models in pathogenesis or preclinical therapeutic studies to follow the accumulation of $3 \mathrm{R}$ and $3 \mathrm{R} / 4 \mathrm{R}$ tau aggregates. Applications to antemortem diagnosis or therapeutic trials in humans will depend largely upon the ability to detect tau seeds in accessible tissue or fluid specimens such as CSF, but such capability remains to be shown.

A key to success in developing RT-QuIC or other seed amplification assays is maximizing the kinetic difference between seeded and unseeded (spontaneous) polymerization of the substrate molecules. In our experience the $\mathrm{K} 12 \mathrm{CFh}$ substrate is readily seeded but is less prone to spontaneous polymerization than the tau fragments that we have used in our previous tau RT-QuIC assays [21, 23, 28, 29]. This feature can improve assay sensitivity and specificity, which may be crucial for detecting aggregates in accessible diagnostic specimens with relatively low tau seed concentrations. Recently we have shown for six different RT-QuIC assays that modification of the main anionic component in the reaction mixture can also help to delay spontaneous fibril formation [23]. In the case of the AD tau RT-QuIC assay, this strategy made it possible to conduct the assay using only the $\mathrm{t306-378}$ fragment in the presence of weakly-hydrated anions $\left(\mathrm{NaBr}, \mathrm{NaI}, \mathrm{NaClO}_{4}\right)$. Thus, overall, we have simplified the amplification of $\mathrm{AD}$ tau aggregates by two methods: (1) by modifying the ionic species present in the reaction mixture of the original AD tau RT-QuIC [23] and (2) by extending the T306-378 fragment $\mathrm{N}$ terminally to include $\mathrm{R} 1$, and $\mathrm{C}$-terminally to residue 400 in this work.

The ability of K12 RT-QuIC to distinguish PiD- from 3R/4R-seeded reactions based on the ThT fluorescence maxima and FTIR spectra of the reaction products suggests that at least some of the distinct conformational features of $\mathrm{PiD}$ and $3 \mathrm{R} / 4 \mathrm{R}$ tau filaments can be imposed on, and propagated by, the $\mathrm{K} 12 \mathrm{CFh}$ tau fragment. The $1618-1633 \mathrm{~cm}^{-1}$ range of the major spectral differences indicated that they likely reflect variations in $\beta$-sheets formed by the polypeptide backbone because, although side-chain vibrations can absorb in this range, they typically make up only about $10 \%$ of the spectral intensity [22]. Nonetheless, the spectral differences in the more sidechain-rich range below $1400 \mathrm{~cm}^{-1}$ are also consistent with conformational differences. The divergent ThT maxima exhibited by the $3 R$ - versus $3 R / 4 R$-seeded K12CFh fibrils might be due to distinct alignments of the ThT fluorophores on fibrils with different amyloid cores and/or to the differential bundling of the fibrils.

Our inability to discriminate between the $3 R / 4 R$ seed subtypes (e.g. AD, CTE and PART) could be because the filament core conformations of $\mathrm{AD}$ and CTE, at least, are closely similar to one another $[15,16]$. Moreover, PART pathology may be on a continuum with the tau pathology of $\mathrm{AD}$, as both involve deposition of $3 \mathrm{R} / 4 \mathrm{R}$ tau isoforms in the entorhinal cortex and hippocampus in neurofibrillary tangles (NFTs); however PART by definition lacks amyloid- $\beta$ pathology $(\mathrm{NFT}+/ \mathrm{A} \beta-)[10,12]$. PART is further defined as having NFT pathology at or below Braak stage IV [12]. Thus, there may be only a quantitative difference between PART and AD tau deposits. This would mean that the low level of seeding activity of PART brains (mean $\operatorname{logSD_{50}} / \mathrm{mg}$ tissue $=4-6$ ) reflects lesser deposition of AD-like tau. Interestingly, 2 of 3 PART samples had seeding levels comparable to that of $4 \mathrm{R}$ tau and IHC tau-negative brain homogenates. This intermediate seeding activity was also observed with the original $\mathrm{AD}$ tau RT-QuIC assay $\left(\operatorname{logSD_{50}} / \mathrm{mg}\right.$ tissue: $\mathrm{KO}<\mathrm{PiD}$ and $4 \mathrm{R}$ tauopathies $<\mathrm{AD}$, Table 1 ) [21], but not in the $3 \mathrm{R}$ or $4 \mathrm{R}$ tau RT-QuIC assays (compare control $\operatorname{logSD_{50}} / \mathrm{mg}$ tissue values in $3 \mathrm{R}$, $4 \mathrm{R}$ tau $\mathrm{RT}$ QuIC columns with KO averages in Table 1) [27, 29]. This seeding activity could therefore be a result of PART-related tau, which has been described elsewhere as nearly universally present at some level in aged brains [6]. NFTs can even be found in the second and third decades of life [7]. Thus, as we have now observed in two assays designed to amplify AD tau filaments (here and [21]), an elevated baseline level of tau seeding activity in control brain specimens may be readily detectable due to similarities of AD and PART tau deposits and the frequent presence of the latter in the brains of clinicallynormal individuals at quantities that may not be detectable by immunohistochemistry.

Pick bodies are rarely seen in AD brains. However, in PiD brains, whereas $3 \mathrm{R}$ tau filaments predominate, tau filaments that contain $4 \mathrm{R}$ tau isoforms have also been observed [37]. The fact that K12 RT-QuIC detects both $\mathrm{PiD}$ - and $\mathrm{AD}$-associated tau seeds raises the issue of how the assay might respond quantitatively to brain samples with mixed tau pathologies. Initially, using K12 RT-QuIC, we estimated an average of $8.3 \log \mathrm{SD}_{50} / \mathrm{mg}$ $\mathrm{AD}$ brain compared to $7.45 \log \mathrm{SD}_{50} / \mathrm{mg} \mathrm{PiD}$ brain (Fig. 3), which suggested a greater deposition of tau seeds in AD brains. This conclusion is difficult to disentangle, however, from the possibility of different seeding efficiencies of structurally distinct tau aggregates [14-16]. For example, our assays of AD- and PiDseeded synthetic fibrils of predetermined concentration revealed an additional log of sensitivity in detecting the PiD-seeded fibrils (7.5 fM vs $75 \mathrm{fM}$, Additional file 6). This suggests that the templating of $\mathrm{K} 12 \mathrm{CFh}$ onto PiDseeded fibrils is more efficient. One possible explanation is that the more tightly bundled AD fibrils may present fewer exposed seeding surfaces for K12CFh incorporation, relative to the loosely dispersed filaments of Pick bodies [31]. Also differences in the bundling or organization of $\mathrm{AD}$ - versus $\mathrm{PiD}$-seeded $\mathrm{K} 12 \mathrm{CFh}$ fibrils 
may impede fragmentation or lateral secondary nucleation to generate new seeding surfaces during RT-QuIC reactions. When we assayed ratios of $\mathrm{AD}: \mathrm{PiD} \mathrm{BHs}$ that each had $\sim 8 \log \mathrm{SD}_{50} / \mathrm{mg}$ (PiD 7, AD 2 in Fig. 3) we expected to see a linear reduction in ThT amplitude as the AD:PiD ratio decreased. Instead, we saw disproportionate drop in ThT fluorescence maximum even a 9:1 AD:PiD ratio (Additional file 5), suggesting a favorability of the K12CFh tau construct to adopt the PiD fold. However, it also remains possible, considering the inherent inaccuracy in endpoint dilution RT-QuIC assays of several fold, that the PiD $\mathrm{BH}$ had a higher seed concentration than the $\mathrm{AD} \mathrm{BH}$. Nonetheless, both the data in Additional file 5 and the additional $\log$ of sensitivity of K12 RT-QuIC for the PiDseeded synthetic fibrils (Additional file 6) are consistent with more efficient seeding of $\mathrm{K} 12 \mathrm{CFh}$ by PiD tau seeds. Further studies on brain tissue with varying amounts of $\mathrm{AD}$ (3R/4R) and PiD (mainly 3R but sometimes 4R) copathology will be needed to fully understand the significance of these observations.

\section{Conclusions}

In summary, we have described a single ultrasensitive assay that detects both $3 \mathrm{R}$ and $3 \mathrm{R} / 4 \mathrm{R}$ tau aggregates and discriminates the seeds of $\mathrm{AD}$ and CTE from those of PiD. This assay simplifies the measurement of these seeds by providing a single test that can substitute for our two previously described 3R (PiD) and 3R/4R (AD) tau RT-QuIC assays, and requires the preparation of one tau substrate, herein called $\mathrm{K} 12 \mathrm{CFh}$, instead of the 2 required for our previous assays. Further, this single K12CFh tau fragment showed less frequent spontaneous fibrillization compared to the substrates used in the AD RT-QuIC assay, improving the confidence with which a true positive can be declared. Importantly, the analytical sensitivity of the assay is consistent with those of the previously-published $\mathrm{AD}$ and PiD tau RT-QuIC assays.

Surprisingly, given the strong selectivity of our previous $3 R$ tau RT-QuIC assay, our results also show that $3 R / 4 R$ tau aggregates can seed the fibrilization of a pure $3 R$ tau fragment that is $\mathrm{C}$-terminally extended. So, from a more fundamental perspective, our biophysical characterization of the products of K12 RT-QuIC provided evidence that at least some features of the original conformations of the aggregates are propagated faithfully in these fibrillization reactions, making the assay a promising tool for further studying the misfolding process or inhibitors thereof.

\section{Supplementary information}

Supplementary information accompanies this paper at https://doi.org/10. 1186/s40478-020-0887-z.

Additional file 1. Purification of $\mathrm{K} 12 \mathrm{CFh}$ tau. Following overnight autoinduction of K12CFh-expressing E. coli, tau is bound to a his column and eluted over a stepwise gradient of $23-100 \%$ buffer B (46-200 mM imidazole). Designated fractions are pooled and precipitated in acetone overnight, washed, dissolved in $8 \mathrm{M} \mathrm{GdnHCl}$ in PBS, and desalted by PD10 size exclusion column. $\mathrm{K} 12 \mathrm{CFh}$ fractions void of $\mathrm{GdnHCl}$ are pooled and stored at $-80^{\circ} \mathrm{C}$ for future use.

Additional file 2. Salt optimization of the K12 tau RT-QulC assay. Panels represent lag time (time to threshold of ThT $>100^{*}$ SD baseline fluorescence) for reactions seeded with serial dilutions of PiD and AD brain tissue in four salts at $400 \mathrm{mM}$. Top: addition of $40 \mu \mathrm{M}$ heparin; bottom: absence of heparin.

Additional file 3. Robustness of ThT amplitude differences with modified reaction conditions. Panels represent mean ThT amplitude data for three PiD and three AD brain homogenate reactions seeded at $1 x$ $10^{-4}$ dilution in $\mathrm{K} 12$ tau RT-QulC. Each panel shows a variation of a reaction condition (A, tau concentration; B, ThT concentration; C, NaF concentration; $D$, addition of silica beads; $E$, heparin concentration; $F$, plate reader gain, G; temperature of ThT read) beyond that of the optimized conditions listed in the figure. For gain and temperature plots, the same reaction at endpoint was scanned over increasing gain and temperature of the plate holder.

Additional file 4. Second derivative FTIR spectra of PiD (3R)-, AD (3R/ $4 R)$ - and CTE (3R/4R)-seeded K12CFh products in fingerprint region from 800 to $1200 \mathrm{~cm}^{-1}$. Each trace represents spectra from eight pooled K12 RT-QulC reaction products seeded with an individual $1 \times 10^{-4}$ dilution of brain homogenate. Multiple traces represent reaction products seeded with brain homogenate from separate individuals: eight PiD (red), three SAD (orange), three fAD (green), and three CTE (blue).

Additional file 5. K12 RT-QulC performance in the presence of $A D$ and PiD tau seeds. Sixteen replicate K12 RT-QulC reactions were performed at the given ratio of AD:PiD seeds.

Additional file 6. Analytical sensitivity of K12 RT-QulC seeded with synthetic K12CFh fibrils. Panels represent lag time analysis (time where ThT fluorescence exceeded $100 *$ SD baseline fluorescence) for eight replicate reactions seeded with the designated synthetic K12CFh fibril concentrations. Assay conditions and dilution buffer were identical to those described in methods.

\section{Abbreviations}

3R: 3-repeat; 4R: 4-repeat; AD: Alzheimer disease; AGD: Argyrophilic grain disease; ALS: Amyotrophic lateral sclerosis; ATR-FTIR: Attenuated total reflectance Fourier-transform infrared spectroscopy; BH: Brain homogenate; CBD: Corticobasal degeneration; CSF: Cerebrospinal fluid; CTE: Chronic traumatic encephalopathy; CVD: Cerebrovascular disease; DLBD: Diffuse Lewy body dementia; fAD: Familial Alzheimer disease; FTDP 17 MAPT: Frontotemporal dementias associated with MAPT mutations; FTLDTDP43: Frontotemporal lobar degeneration with TDP43 protein; GdnHCl: Guanidine-HCl; IVS10 + 3G > A FTDP: Frontotemporal dementia with parkinsonism; K12CFh: K12 cysteine-free +6x His; K19CFh: K19 cysteine-free +6x His; KO: Tau knock out (tau-free); MSA: Multiple system atrophy; NaF: Sodium fluoride; PART: Primary age-related tauopathy; PBS: Phosphate buffered saline; PD: Parkinson's disease; PiD: Pick disease; PSP: Progressive supranuclear palsy; RFU: Relative fluorescence units; RT-QulC: Real-time quaking induced conversion; SAD: Sporadic Alzheimer disease; SC: Senile change; TEM: Transmission electron microscopy; ThT: Thioflavin T

\section{Acknowledgements}

We gratefully acknowledge Drs. Brent Race, Alyssa Evans, Bradley Groveman, and Moses Leavens for their thoughtful feedback on this manuscript. We thank Dr. David Dorward and Cindi Schwartz for assistance with electron microscopy.

\section{Authors' contributions}

MM: mainly conceived the study, and performed experimental design and assay optimization, recombinant K12CFh purification, FTIR analysis, logSD $\mathrm{S}_{50}$ determinations, manuscript preparation and revision; NCF: recombinant K12CFh purification, $\operatorname{logSD}_{50}$ determinations, manuscript preparation and revision; ES: designed the K12CFh construct and initially helped conceive the study; AK: TEM grid preparation, manuscript preparation; BG, KN, GZ: diagnosed the cases neuropathologically, provided the tissue and neuropathologic insights for discussion, and revised the manuscript.; MV: 
manuscript preparation and revision; BC: manuscript preparation and revision, experimental design and oversight. All authors read and approved the final manuscript.

\section{Authors' information}

ES is currently at UCB Biopharma SPRL, Chemin du Foriest B-1420 BraineI'Alleud, Belgium. AK is currently at the Department of Pathology, Case Western Reserve University School of Medicine, Cleveland, $\mathrm{OH}$.

\section{Funding}

This work is supported in part by the Intramural Research Program of the NIAID (to BC); MM is supported by the NIH/Cambridge scholars program; BG and KN are supported by grant PHS P30-AG010133; a Creutzfeldt-Jakob Disease Foundation grant supported GZ; a Japan Society for the Promotion of Science Fellowship (JSPS) for Japanese Biochemical and Behavioral Researchers at NIH supported ES. We acknowledge the Massachusetts Alzheimer's Disease Research Center (P50 AG005134).

\section{Availability of data and materials}

The datasets supporting the conclusions of this article are included within the article (and its additional file(s)).

\section{Ethics approval and consent to participate}

All brain samples were collected post-mortem obtained from sources indicated in Online Resource Table 1 and Acknowledgements in [17] and deidentified prior to RT-QulC testing.

\section{Consent for publication}

Not applicable given that the brain samples were collected post-mortem and de-identified.

\section{Competing interests}

MAM, ES, AK and BC are named as inventors on patent applications pertaining to the tau RT-QulC methodologies.

\section{Author details}

'LPVD, Rocky Mountain Laboratories, NIAID, NIH, Hamilton, MT 59840, USA. ${ }^{2}$ Centre for Misfolding Diseases, Department of Chemistry, University of Cambridge, Cambridge CB2 1EW, UK. ${ }^{3}$ Department of Pathology and Laboratory Medicine, Indiana University School of Medicine, Indianapolis, IN 46202, USA. ${ }^{4}$ Department of Neurosciences, University of Verona, 37129 Verona, Italy.

Received: 18 December 2019 Accepted: 26 January 2020

Published online: 22 February 2020

\section{References}

1. Atarashi R, Moore RA, Sim VL, Hughson AG, Dorward DW, Onwubiko HA, Priola SA, Caughey B (2007) Ultrasensitive detection of scrapie prion protein using seeded conversion of recombinant prion protein. NatMethods 4:645-650

2. Atarashi R, Satoh K, Sano K, Fuse T, Yamaguchi N, Ishibashi D, Matsubara T, Nakagaki T, Yamanaka H, Shirabe S et al (2011) Ultrasensitive human prion detection in cerebrospinal fluid by real-time quaking-induced conversion. Nat Med 17:175-178. https://doi.org/10.1038/nm.2294

3. Atarashi R, Wilham JM, Christensen L, Hughson AG, Moore RA, Johnson LM, Onwubiko HA, Priola SA, Caughey B (2008) Simplified ultrasensitive prion detection by recombinant PrP conversion with shaking. NatMethods 5:211-212

4. Bongianni M, Ladogana A, Capaldi S, Klotz S, Baiardi S, Cagnin A, Perra D, Fiorini M, Poleggi A, Legname G et al (2019) alpha-Synuclein RT-QulC assay in cerebrospinal fluid of patients with dementia with Lewy bodies. Ann Clin Transl Neurol 6:2120-2126. https://doi.org/10.1002/acn3.50897

5. Bongianni M, Orrù CD, Groveman BR, Sacchetto L, Fiorini M, Tonoli G, Triva G, Capaldi S, Testi S, Ferrari S et al (2017) Diagnosis of Human Prion Disease Using Real-Time Quaking-Induced Conversion Testing of Olfactory Mucosa and Cerebrospinal Fluid Samples. JAMA Neurology 74:1-8. https://doi.org/ 10.1001/jamaneurol.2016.4614

6. Bouras C, Hof PR, Morrison JH (1993) Neurofibrillary tangle densities in the hippocampal formation in a non-demented population define subgroups of patients with differential early pathologic changes. Neurosci Lett 153. https://doi.org/10.1016/0304-3940(93)90305-5

7. Braak H, Del Tredici K (2011) The pathological process underlying Alzheimer's disease in individuals under thirty. Acta Neuropathol 121. https://doi.org/10.1007/s00401-010-0789-4

8. Candelise N, Schmitz M, Llorens F, Villar-Pique A, Cramm M, Thom T, da Silva Correia SM, da Cunha JEG, Mobius W, Outeiro TF et al (2019) Seeding variability of different alpha synuclein strains in synucleinopathies. Ann Neurol 85:691-703. https://doi.org/10.1002/ana.25446

9. Colby DW, Wain R, Baskakov IV, Legname G, Palmer CG, Nguyen HO, Lemus A, Cohen FE, DeArmond SJ, Prusiner SB (2010) Protease-sensitive synthetic prions. PLoS Pathog 6:e1000736. https://doi.org/10.1371/journal.ppat. 1000736

10. Crary JF, Trojanowski JQ, Schneider JA, Abisambra JF, Abner EL, Alafuzoff I, Arnold SE, Attems J, Beach TG, Bigio EH et al (2014) Primary age-related tauopathy (PART): a common pathology associated with human aging. Acta Neuropathol 128:755-766. https://doi.org/10.1007/s00401-014-1349-0

11. Dougherty RM (1964) Animal virus titration techniques. In: Harris RJC (ed) Techniques in experimental virology. Academic Press, Inc., City, pp 183-186

12. Duyckaerts C, Braak H, Brion JP, Buee L, Del Tredici K, Goedert M, Halliday G, Neumann M, Spillantini MG, Tolnay M et al (2015) PART is part of Alzheimer disease. Acta Neuropathol 129:749-756. https://doi.org/10.1007/s00401-0151390-7

13. Fairfoul G, McGuire LI, Pal S, Ironside JW, Neumann J, Christie S, Joachim C, Esiri M, Evetts SG, Rolinski M et al (2016) Alpha-synuclein RT-QulC in the CSF of patients with alpha-synucleinopathies. Ann Clin Transl Neurol 3:812-818. https://doi.org/10.1002/acn3.338

14. Falcon B, Zhang W, Murzin AG, Murshudov G, Garringer HJ, Vidal R, Crowther RA, Ghetti B, Scheres SHW, Goedert M (2018) Structures of filaments from Pick's disease reveal a novel tau protein fold. Nature 561: 137-140. https://doi.org/10.1038/s41586-018-0454-y

15. Falcon B, Zivanov J, Zhang W, Murzin AG, Garringer HJ, Vidal R, Crowther RA, Newell KL, Ghetti B, Goedert M et al (2019) Novel tau filament fold in chronic traumatic encephalopathy encloses hydrophobic molecules. Nature 568:420-423. https://doi.org/10.1038/s41586-019-1026-5

16. Fitzpatrick AWP, Falcon B, He S, Murzin AG, Murshudov G, Garringer HJ, Crowther RA, Ghetti B, Goedert M, Scheres SHW (2017) Cryo-EM structures of tau filaments from Alzheimer's disease. Nature 547:185-190. https://doi. org/10.1038/nature23002

17. Friedhoff $P$, von Bergen M, Mandelkow EM, Davies P, Mandelkow E (1998) A nucleated assembly mechanism of Alzheimer paired helical filaments. Proc Natl Acad Sci U S A 95:15712-15717

18. Green AJE, Zanusso G (2018) Prion protein amplification techniques. Handb Clin Neurol 153:357-370. https://doi.org/10.1016/B978-0-444-63945-5.00019-2

19. Groveman BR, Orru CD, Hughson AG, Raymond LD, Zanusso G, Ghetti B, Campbell KJ, Safar J, Galasko D, Caughey B (2018) Rapid and ultra-sensitive quantitation of disease-associated alpha-synuclein seeds in brain and cerebrospinal fluid by alphaSyn RT-QuIC. Acta Neuropathol Commun 6:7. https://doi.org/10.1186/s40478-018-0508-2

20. Jack CR, Bennett DA, Blennow K, Carrillo MC, Dunn B, Haeberlein SB, Holtzman DM, Jagust W, Jessen F, Karlawish J et al (2018) NIA-AA Research Framework: Toward a biological definition of Alzheimer's disease. Alzheimers Dement 14:535-562. https://doi.org/10.1016/j.jalz. 2018.02.018

21. Kraus A, Saijo E, Metrick MAl, Newell K, Sigurdson C, Zanusso G, Ghetti B, Caughey B (2019) Seeding selectivity and ultrasensitive detection of tau aggregate conformers of Alzheimer disease. Acta Neuropathol 137:585-598. https://doi.org/10.1007/s00401-018-1947-3

22. Krimm S, Bandekar J (1986) Vibrational spectroscopy and conformation of peptides, polypeptides, and proteins. AdvProtein Chem 38:181-364

23. Metrick MA 2nd, do Carmo Ferreira N, Saijo E, Hughson AG, Kraus A, Orru C, Miller MW, Zanusso G, Ghetti B, Vendruscolo M et al (2019) Million-fold sensitivity enhancement in proteopathic seed amplification assays for biospecimens by Hofmeister ion comparisons. Proc Natl Acad Sci U S A. https://doi.org/10.1073/pnas.1909322116

24. Orru CD, Groveman BR, Raymond LD, Hughson AG, Nonno R, Zou W, Ghetti B, Gambetti P, Caughey B (2015) Bank Vole Prion Protein As an Apparently Universal Substrate for RT-QulC-Based Detection and Discrimination of Prion Strains. PLoS Path 11:e1004983. https://doi.org/10.1371/journal.ppat.1004983

25. Orru CD, Soldau K, Cordano C, Llibre-Guerra J, Green AJ, Sanchez H, Groveman BR, Edland SD, Safar JG, Lin JH et al (2018) Prion Seeds Distribute 
throughout the Eyes of Sporadic Creutzfeldt-Jakob Disease Patients. MBio 9. https://doi.org/10.1128/mBio.02095-18

26. Orru CD, Wilham JM, Raymond LD, Kuhn F, Schroeder B, Raeber AJ, Caughey B (2011) Prion disease blood test using immunoprecipitation and improved quaking-induced conversion. mBio 2:e00078-e00011. https://doi. org/10.1128/mBio.00078-11

27. Saijo E, Ghetti B, Zanusso G, Oblak A, Furman JL, Diamond MI, Kraus A, Caughey B (2017) Ultrasensitive and selective detection of 3-repeat tau seeding activity in Pick disease brain and cerebrospinal fluid. Acta Neuropathol 133:751-765. https://doi.org/10.1007/s00401-017-1692-z

28. Saijo E, Groveman BR, Kraus A, Metrick M, Orru CD, Hughson AG, Caughey B (2019) Ultrasensitive RT-QulC Seed Amplification Assays for DiseaseAssociated Tau, alpha-Synuclein, and Prion Aggregates. Methods Mol Biol 1873:19-37. https://doi.org/10.1007/978-1-4939-8820-4_2

29. Saijo E, Metrick MA 2nd, Koga S, Parchi P, Litvan I, Spina S, Boxer A, Rojas JC, Galasko D, Kraus A et al (2019) 4-Repeat tau seeds and templating subtypes as brain and CSF biomarkers of frontotemporal lobar degeneration. Acta Neuropathol. https://doi.org/10.1007/s00401-019-02080-2

30. Shahnawaz M, Tokuda T, Waragai M, Mendez N, Ishii R, Trenkwalder C, Mollenhauer B, Soto C (2017) Development of a Biochemical Diagnosis of Parkinson Disease by Detection of alpha-Synuclein Misfolded Aggregates in Cerebrospinal Fluid. JAMA Neurol 74:163-172. https://doi.org/10.1001/ jamaneurol.2016.4547

31. Takauchi S, Hosomi M, Marasigan S, Sato M, Hayashi S, Miyoshi K (1984) An ultrastructural study of Pick bodies. Acta Neuropathol 64:344-348. https:// doi.org/10.1007/bf00690400

32. Wang Z, Manca M, Foutz A, Camacho MV, Raymond GJ, Race B, Orru CD, Yuan J, Shen P, Li B et al (2019) Early preclinical detection of prions in the skin of prion-infected animals. Nat Commun 10:247. https://doi.org/10.1038/ s41467-018-08130-9

33. Wilham JM, Orrú CD, Bessen RA, Atarashi R, Sano K, Race B, Meade-White KD, Taubner LM, Timmes A, Caughey B (2010) Rapid End-Point Quantitation of Prion Seeding Activity with Sensitivity Comparable to Bioassays. PLoS Path 6:e1001217. https://doi.org/10.1371/journal.ppat.1001217

34. Wille H, Drewes G, Biernat J, Mandelkow EM, Mandelkow E (1992) Alzheimer-like paired helical filaments and antiparallel dimers formed from microtubule-associated protein tau in vitro. J Cell Biol 118:573-584. https:// doi.org/10.1083/jcb.118.3.573

35. Zanusso G, Monaco S, Pocchiari M, Caughey B (2016) Advanced tests for early and accurate diagnosis of Creutzfeldt-Jakob disease. Nat Rev Neurol 12:325-333. https://doi.org/10.1038/nrneurol.2016.65

36. Zhang K, Tarutani A, Newell KL, Murzin AG, Matsubara T, Falcon B, Vidal R, Garringer HJ, Shi Y, Ikeuchi T et al (2019) Novel tau filament fold in corticobasal degeneration, a four-repeat tauopathy. bioRxiv. https://doi.org/ $10.1101 / 811703$

37. Zhukareva V, Mann D, Pickering-Brown S, Uryu K, Shuck T, Shah K, Grossman M, Miller BL, Hulette CM, Feinstein SC et al (2002) Sporadic Pick's disease: a tauopathy characterized by a spectrum of pathological tau isoforms in gray and white matter. Ann Neurol 51:730-739. https://doi.org/10.1002/ana. 10222

\section{Publisher's Note}

Springer Nature remains neutral with regard to jurisdictional claims in published maps and institutional affiliations.

Ready to submit your research? Choose BMC and benefit from:
- fast, convenient online submission
- thorough peer review by experienced researchers in your field
- rapid publication on acceptance
- support for research data, including large and complex data types
- gold Open Access which fosters wider collaboration and increased citations
- maximum visibility for your research: over 100M website views per year
At BMC, research is always in progress.
Learn more biomedcentral.com/submissions

